\title{
Is the Principia publishable now?
}

\author{
Newton's theory of gravitation was a landmark in science but might easily have fallen foul of modern referees \\ demanding explanations.
}

NEWTON's theory of gravitation is one of the intellectual wonders of the world. Everybody knows the tale. Kepler's careful brooding over observations of the planets, notably those of Tycho Brahe, had led him to various generalizations about the characteristics of planetary orbits. Seeking the cause of these regularities, Newton came to the gravitational explanation - that each planet is bound to the Sun by a gravitational force inversely proportional to the square of the distance between them. The constant of proportionality is the product of the masses of the Sun and the planet with the universal constant $G$.

Because the dynamical response of a planet to the gravitational force is inversely proportional to its mass, the shape of a planetary orbit is independent of the mass of the planet, depending only on the object's specific angular momentum (angular momentum per unit mass). Newton went on to invent a rudimentary form of the differential calculus to demonstrate that closed planetary orbits are ellipses. He and others quickly used the gravitational law to deal with the Moon, and to show that spinning bodies such as the Earth should be flattened at the poles.

This recapitulation of the well known is partly stimulated by the appearance of the latest book by Subramaniam Chandrasekhar, the Nobel prize-winning theoretical astrophysicist and long-term editor of the Astrophysical Journal, who has worked through Newton's Principia, translating the great man's mathematics into modern form (see David Hughes' review on page 395 of this issue).

With the benefit of huge hindsight, we naturally regard the Principia as a landmark in the development of science, which it was. Even the controversy between the supporters of Newton and Leibnitz over proper attribution of priority for the invention of the calculus has long since been forgotten; the records show that the two men had reached the same result independently. And it was Newton, in any case, who for the first time applied the calculus to a problem that really mattered. The intuition that led him to the notion that an inverse square law would make ellipses of planetary orbits is nevertheless beyond ordinary ken.

Yet there is a flaw in the gravitational law at which Christiaan Huyghens, Newton's contemporary and intellectual equal, among others protested. What is all this about action at a distance? After all, Newton's law assumed that the gravitational force was transmitted between a planet and the Sun without the intervention of any medium. By the same test, the force was transmitted instantaneously; increase the distance between a planet and the Sun and the attractive force is instantly decreased.

This aspect of the law of gravitation is plainly unphysical in the modern sense, but so it was before Newton's time. Had not the Ancients (Aristotle included) shrunk from the idea that a projectile could continue to move through the air without the continued intervention of some external influence? That is why people then invoked the idea that the movement of the projectile created a vacuum into which air would rush, impelling the projectile forward in its track. But Newton's first law deals magisterially with that issue: "a body will continue to move...".

Huyghens, being a Cartesian, had no doubt where matters stood on the law of gravitation. The idea of action at a distance could only be a kind of magic. Forces were transmitted from one object to another only on contact. So what would have happened if, in due course, the Royal Society had sent the manuscript of the Principia out to referees in the modern manner? The chances are that the exercise would have recruited a good many quizzical referees' reports. Anybody can write the dialogue; "by what means, pray, does the author fancy that this magic can be contrived over the great distance between the Sun and Jupiter and without the lapse of time?"

The other issue on which Newton and Huyghens were at odds is not irrelevant. Newton's corpuscular theory of light, the notion that light consists of "photons", was already public knowledge when the first edition of the Principia appeared. Indeed, Newton's contemporary fame rested more directly on his recovery of all the colours of the rainbow from a single beam of white light; that cannot have failed to enhance the reputation of the corpuscular theory.

Famously, Huyghens argued for the wave theory of light propagation. His demonstration of how a propagating wavefront can be constructed from wavelets emitted from the surface at an earlier time has a thoroughly modern ring. There are relics of it in most accounts of wave dispersion. But Huyghens was also vindicated (late in the eighteenth century) by the recognition that his expression for the index of refraction on going from one medium to another was, unlike Newton's, correct.

Certainly by the time Maxwell set about putting electrodynamics on a mathematical basis in the $1860 \mathrm{~s}$, there was no doubt in his mind that action at a distance had to be avoided like the plague. In a paper published at the end of 1864, Maxwell referred to the earlier attempts by Wilhelm Weber to construct a mathematical theory of electromagnetism based on the assumption that there are particles with electrical and magnetic properties "which have the property of acting on one another at a distance by attraction or repulsion".

Dryly, Maxwell notes that Weber had been forced to suppose that the electrostatic force between two charges would depend on their relative velocity: he then declares "I have therefore preferred to seek an explanation ... in another direction". That direction was of course the electromagnetic field which Maxwell took to be the source of electrical and magnetic forces. Following the familiar argument due to Huyghens, he concluded that there is an "æotherial medium filling space and permeating bodies and capable of being set in motion and of transmitting that motion from one part to another". That of course was the "luminiferous æther", the search for which occupied many of Maxwell's successors for much of the nineteenth century. In the end, the lumeniferous æther collapsed under the weight of its internal contradictions.

Now, of course, Newton's corpuscular theory of light enjoys as much standing as Huyghens' account of wave motion in empty space. They are complementary aspects of light in the sense of quantum mechanics. It is nevertheless sobering that the sharp contrast of style between Newton and Huyghens has only now been resolved by the redefinition, in the language of quantum mechanics, of what the vacuum is.

Empty space is, in principle, a potential source both of photons and of pairs of material particles, electrons for example. Of course, there are strict rules to ensure that neither energy nor momentum appears without good reason, but at least for electromagnetic phenomena, a vacuum is a better version of the luminiferous æther than anybody at the end of the nineteenth century ever hoped to find. Meanwhile, it is intriguing but also important that the sharp conflict over action at a distance three centuries ago is one whose resolution depends crucially on quantum mechanics. Is it at all possible that a perceptive analysis of what, after all, was an honest conflict would have arrived at the truth about the nature of the vacuum much earlier?

John Maddox 